\title{
Memory for the Order of Events in Specific Sequences: Contributions of the Hippocampus and Medial Prefrontal Cortex
}

\author{
Loren M. DeVito and Howard Eichenbaum \\ Center for Memory and Brain, Boston University, Boston, Massachusetts 02215
}

\begin{abstract}
Episodic memory involves remembering the incidental order of a series of events that comprise a specific experience. Current models of temporal organization in episodic memory have demonstrated that animals can make memory judgments about the order of serially presented events; however, in these protocols, the animals can judge items based on their relative recency. Thus, it remains unclear as to whether animals use the specific order of items in forming memories of distinct sequences. To resolve this important issue in memory representation, we presented mice repeatedly with two widely separated odor sequences and then tested their natural exploratory preference between pairs of odors selected from within or between sequences. Intact animals preferred to investigate odors that occurred earlier within each sequence, indicating they did remember the order of events within each distinct sequence. In contrast, intact animals did not discriminate between pairs of odors from different sequences. These findings indicate that preferences were not guided by relative recency, which would be expected to support graded discrimination between widely separated events. Furthermore, damage to either the hippocampus or the medial prefrontal cortex eliminated order preference within sequences. Despite the deficit in order memory, control recognition tests showed that normal mice and mice with hippocampal or medial prefrontal damage could correctly identify previously experienced odors compared with novel odors. These findings provide strong evidence that animals form representations of the order of events within specific experiences and that the hippocampus and prefrontal cortex are essential to order memory.
\end{abstract}

\section{Introduction}

Episodic memory requires the binding of sequential experiences, such that memories are organized by the order of occurrence of serial events (Tulving and Markowitsch, 1998). Memory for sequential events structures the flow of our daily life, and the ability to integrate events that occur across different points in time is a crucial requisite for the later retrieval of experiences (Clayton and Dickinson, 1998; Eichenbaum, 2004). A few studies have explored memory for sequential events in specific episodes (Chiba et al., 1997; Fortin et al., 2002; Kesner et al., 2002). Fortin et al. (2002) and Kesner et al. (2002) presented rats with unique sequences of five odors and trained them to judge the earlier presented of two selected odors, such that animals gradually learned the rule to choose an item that had been experienced less recently over one that was experienced more recently. Memory for sequential events has also been evaluated using an object recognition paradigm that exploits animals' natural (untrained) tendency to explore a less recently experienced object compared with a more recently experienced object (Dere et al., 2005).

\footnotetext{
Received Aug. 11, 2010; revised Dec. 22, 2010; accepted Dec. 29, 2010.

This study was supported by the Conte Center for Schizophrenia Research/National Institutes of Health Grant MH60450. Thanks to Christine Lykken for assistance with processing and analyzing histology. Parts of this paper have been published previously at the Society for Neuroscience 2009 meeting.

Correspondence should be addressed to Dr. Howard Eichenbaum, Center for Memory and Brain, Boston University, Boston, MA 02215. E-mail: hbe@bu.edu.

DOI:10.1523/JNEUROSCI.4202-10.2011

Copyright $\odot 2011$ the authors $\quad 0270-6474 / 11 / 313169-07 \$ 15.00 / 0$
}

Experiments using these protocols have indicated that animals can judge the order of previously experienced events. However, in these tasks, accurate performance may be based on judgments about relative recency of serial experiences, perhaps by using a comparison of differential memory strengths for more and less recently experienced events. Indeed, one of these studies showed that the memory strengths of serially presented odors did differ according to their recency (Fortin et al., 2002). Therefore, these studies do not provide definitive evidence that animals solve these tasks using a direct representation of the order of events within each specific experience.

To address this important issue, we developed a novel behavioral protocol that, as in natural episodic memory, assesses the ability of animals to remember the order of sequential events in specific experiences without explicit training on order judgments. Our design involved a large separation between exposure to multiple sequences and subsequent memory testing on those sequences, to reduce relative differences in memory strengths among the events. Furthermore, we compared performance on different tests that measure memory for order within a particular sequence versus memory for the relative recency of items that occurred at different times within each day.

Previous experiments that have investigated the neural substrates of sequence memory have indicated that both the hippocampus and prefrontal cortex play an important role (Mitchell and Laiacona, 1998; Agster et al., 2002; Fortin et al., 2002; Barker et al., 2007). Therefore, we evaluated the effects of selective lesions of the hippocampus or prefrontal cortex using a newly de- 
veloped protocol that distinguishes memory for temporal organization in specific sequences of events, memory for the relative recency of events that are experienced across different sequences, and memory for the identity of items that appear in these sequences.

\section{Materials and Methods}

Subjects. Male C57BL/6 mice were purchased from the Charles River Laboratory. All animals were maintained on a reverse $12 \mathrm{~h}$ light/dark cycle (lights off at 9:00 A.M., lights on at 9:00 P.M.). Animals were given ad libitum access to food and water, unless otherwise specified in the behavioral methods. Sixty animals were used in this study: 24 served as controls for verification of the task, 8 animals received lesions of the hippocampus, 10 animals received lesions of the medial prefrontal cortex, 8 served as sham-operated medial prefrontal controls, and 10 served as sham-operated hippocampal controls. The Institutional Animal Care and Use Committee of Boston University approved the treatment and use of the animals in these experiments.

Surgery. Bilateral lesions of the hippocampus were made using NMDA $(10 \mathrm{mg} / \mathrm{ml}$; Sigma $)$ or sterile PBS for sham operations, delivered via a microinfusion pump connected to a $5 \mu$ l Hamilton syringe. Animals were anesthetized with a ketamine/xylazine mixture $(0.01 \mathrm{ml} / \mathrm{g})$, and diazepam $(0.02 \mathrm{ml})$ was administered preoperatively to prevent seizures. After the animal had been placed into a stereotaxic head frame, the skull was exposed and the coordinates of bregma were measured. The skull overlying the four coordinates was drilled and dura was removed. Before infusions were made, the syringe was lowered $0.2 \mathrm{~mm}$ for the first two coordinates (dorsal hippocampus) and $0.5 \mathrm{~mm}$ for the last two coordinates (ventral hippocampus) past the injection site and kept a lower depth for $1 \mathrm{~min}$ to increase spread of drug diffusion. The syringe was then raised to the injection site, and the drug was infused over a $2 \mathrm{~min}$ period ( $3 \mathrm{~min}$ infusion for the last coordinate). The needle was left in place for another 5 min before being slowly withdrawn. The complete dorsal and ventral hippocampus was targeted (including the CA fields, dentate gyrus, and subiculum) at four stereotaxic coordinates: anteroposterior (AP) +1.7 , mediolateral $(\mathrm{ML}) \pm 1.2$, dorsoventral $(\mathrm{DV})-1.5 ; \mathrm{AP}+2.3$, $\mathrm{ML} \pm 1.75, \mathrm{DV}-1.75 ; \mathrm{AP}+2.8, \mathrm{ML} \pm 3, \mathrm{DV}-3$; $\mathrm{AP}+3.1, \mathrm{ML} \pm 2.85$, $\mathrm{DV}-3.75$. Fifty nanoliters was infused into the first three sites, and $75 \mathrm{nl}$ was infused into the fourth site. Animals that received sham operations were administered the same procedures as the lesion groups, except that PBS was infused into each site instead of NMDA.

Bilateral lesions of the medial prefrontal cortex were made using ibotenic acid ( $0.06 \mathrm{M}$; Tocris Cookson), or sterile saline for sham operations, delivered via a microinfusion pump connected to a $10 \mu$ l Hamilton syringe attached to a pulled microglass pipette tip. Animals were anesthetized with a ketamine/xylazine mixture $(0.01 \mathrm{ml} / \mathrm{g})$, and diazepam $(0.02 \mathrm{ml})$ was administered preoperatively to prevent seizures. After the animal had been placed into a stereotaxic head frame, the skull was exposed and the coordinates of bregma were measured (the mediolateral values were taken at the level of the midsagittal vein and not at bregma). The skull overlying the two coordinates was drilled and dura was removed. The syringe was lowered to the injection site, and the drug was infused over a $5 \mathrm{~min}$ period. The needle was left in place for another $5 \mathrm{~min}$ before being slowly withdrawn. The infralimbic and prelimbic cortices were targeted at two stereotaxic coordinates: AP $-2.1, \mathrm{ML} \pm 0.25$, DV -2.3 . One hundred fifty nanoliters were infused into both sites. Animals that received sham operations were administered the same procedures as the lesion groups, except that PBS was infused into each site instead of NMDA.

After all infusions, the scalp was sutured, the animal was given $0.4 \mathrm{ml}$ of lactated Ringer's solution to hydrate, and the animal was placed next to a hot water bottle to return body temperature to normal. After surgery, the animal received Children's Tylenol in its water and was provided with soft food and Nutrical. Each animal was allowed 2 weeks to recover before behavioral testing.

Behavioral methods. Mice were tested on a novel protocol that assessed the ability to remember the order of odors presented within specific sequences or across different sequences. Animals were placed on food
A Days 1-5: Sequence Presentation Days
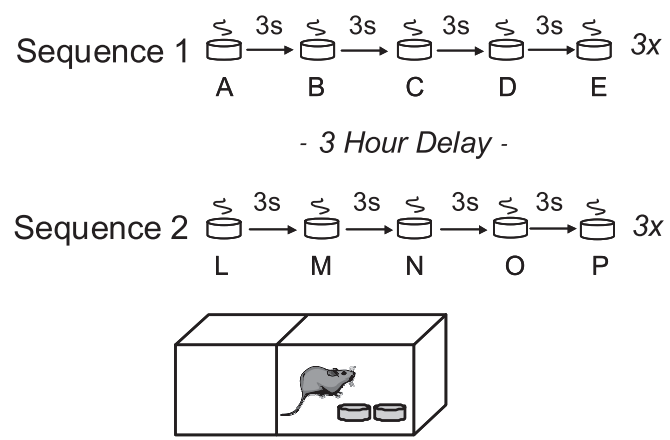

B Days 6-9: Probe Test Days

\begin{tabular}{llcc} 
Within Sequence & $\begin{array}{c}\text { Between Sequence } \\
\text { Day } 6\end{array}$ & $\begin{array}{c}\text { Novelty } \\
\text { Day } 7\end{array}$ & $\frac{\underline{D} \text { Day } 8}{\text { Day } 9}$ \\
\hline L vs. P & A vs. D & Avs. N & L vs. X A vs. X \\
C vs.D & N vs. P & Evs. O & C vs. X N vs. X \\
M vs.O & B vs. C & B vs. L & M vs. X B vs. X
\end{tabular}

Figure 1. Odor sequence task. $A$, Schematic of the odors and testing apparatus used in odor presentation. $\boldsymbol{B}$, Probe tests used to assess memory for sequential order.

restriction and maintained at $85 \%$ of free-feeding weight. Over a $3 \mathrm{~d}$ period, animals were allowed to dig for chocolate sprinkle rewards buried in sand that filled small plastic cups in a Plexiglas mouse cage $(26 \mathrm{~cm}$ length $\times 15 \mathrm{~cm}$ width $\times 13 \mathrm{~cm}$ height) (Fig. $1 \mathrm{~A}$ ). Once they were reliably digging, the animals were exposed to two different odor sequences each day over a $5 \mathrm{~d}$ period. All odors are prepared at $1 \%$ concentration by weight in sand.

Sequence 1, with the odors referred to as A, B, C, D, and E, consisted of the following odors: A, cilantro (Spice Islands Trading Company); B, marjoram (Frontier Natural Products Co-op); C, dill (Frontier Natural Products); D, parsley (Frontier Natural Products Co-op); and E, allspice (Spice Islands Trading Company). Sequence 2, referred to as L, M, N, O, and $\mathrm{P}$, consisted of the following odors: L, lemon (McCormick); M, cinnamon (McCormick); N, tarragon (Frontier Natural Products Coop); $\mathrm{O}$, anise (McCormick), and $\mathrm{P}$, orange (McCormick) (Fig. $1 A$ ).

In the first session each day, the animal was presented with sequence 1 . At the beginning of each sequence, the animal was presented with the first odor, which contained a buried chocolate sprinkle that the mouse could obtain by digging in the sand (Fig. $1 A$ ). This process was repeated for the remaining four odors in that sequence until the animal had been exposed to all five odors successively. Each animal was presented with sequence 1 three times. Three hours after the first session was completed, the animals were exposed to sequence 2 three times in the same manner. This procedure for sequence presentation was repeated for $5 \mathrm{~d}$. After these $5 \mathrm{~d}$, the animals were not subsequently exposed to the odor sequences.

Beginning the next day, the animals were administered probe tests that assessed memory for the odors within and between each of the sequences (Fig. $1 B$ ). On each of these probe tests, two odors were selected, and both were presented without buried rewards. We measured the amount of time the animals spent digging in each cup, and the two digging times were used to calculate a preference index (PI) (Bunsey and Eichenbaum, 1996); PI = (time digging in the odor that occurred earlier in the sequence - time spent digging in the odor that occurred later in the sequence)/total time digging in both odors.

On the first two days of probe testing (days 6 and 7), the animals were presented with odors selected from the same sequence in which they were presented earlier. These were considered "within-sequence" probe tests because they evaluated the ability of the animals to remember the order of odors that were presented within one of the specific sequences. On the first day of probe testing (day 6), the animals were presented with $\mathrm{L}$ versus $\mathrm{P}, \mathrm{C}$ versus $\mathrm{D}$, and $\mathrm{M}$ versus $\mathrm{O}$. On the second day of probe testing 
(day 7), the animals were presented with $A$ versus $D, N$ versus $P$, and $B$ versus $\mathrm{C}$.

On the third day of probe testing (day 8), the animals were presented with pairs consisting of one odor from sequence 1 and one odor from sequence 2 . These were considered "between-sequence" probe tests because they evaluated the ability of the animals to compare the times at which the odors were presented over the course of each day, i.e., earlier in sequence 1 versus $3 \mathrm{~h}$ later in sequence 2 . The animals were presented with $A$ versus $N$, E versus $O$, and $B$ versus $L$.

Finally, on the fourth day (day 9), the animals were presented with an odor from either sequence against a novel odor they had never experienced. These were considered "novelty" probe tests because they evaluated the ability of the animals to recognize novel compared with familiar odors. The animals were presented with $\mathrm{L}$ versus caraway (McCormick), $\mathrm{C}$ versus mace (McCormick), and $\mathrm{M}$ versus sage (McCormick). After a $3 \mathrm{~h}$ delay, the animals were presented with A versus baby powder (Shaw's Supermarkets), N versus onion (McCormick), and B versus strawberry (Nestlé).

Cohorts of four mice, containing two sham and two lesioned mice, were trained and tested within each $9 \mathrm{~d}$ consecutive testing sequence, allowing us to ensure that each animal received a 3 min delay between each of the three sequence presentations of sequence 1 in the first session of the day and sequence 2 in the second session of the day. For each cohort, training on sequence 1 began at 12:00 P.M., and training on sequence 2 began at 3:00 P.M. The presentation of each odor was done as rapidly as possible such that, for each odor, the animal was allowed to enter the testing area, dig in the scented cup, retrieve the reward, and immediately exit, with $\sim 3$ s between odor presentations during which the investigator switched the scented cups.

A cardboard divider was used to separate the compartment into two areas of equal width, a testing compartment and a waiting compartment. At the start of each session, the animal would be placed into the waiting compartment and a trial would be initiated by lifting the divider, allowing the animal to run into the testing compartment. After each trial, the divider would again be lifted by the experimenter, allowing the animal to run back into the other compartment and wait for the next trial to be initiated. The apparatus was also covered to prevent mice from escaping the test box during the session.

During probe trial sessions, each animal was allowed to dig freely in each cup; neither of the cups was baited. The animal was always presented with the cups in six possible locations within the apparatus during the first $5 \mathrm{~d}$ to prevent the development of spatial biases. The positions of the cups were randomized each day for all trials and were different for each mouse. On days in which probe trials were administered, the locations of the cups were pseudorandomly selected so that each mouse experienced the cups in different locations and cup location was not repeated throughout the session. The time spent digging in each cup was recorded using a stopwatch, and these times were later used to calculate preference indices. To prevent digging behavior from extinguishing, immediately after each probe trial, the animal was presented with a plain cup of sand (with no odor) with chocolate sprinkles that the animal was allowed to eat. With the exception of the novelty probe trials on day 4 of testing in which each animal received six probe trials, each animal received three probe trials per day.

To determine whether the preferences reported in the results could have been attributable to innate biases toward or against particular odors, a separate group of eight naive mice were shaped to dig in cups, just as the trained groups described above. Then, each animal was immediately administered the same set of probe trials without exposure to the sequences. Furthermore, to account for potential differences in performance on within-sequence and between-sequence probes attributable to the order in which these probe trials were administered, two additional separate groups of eight mice were shaped to dig in cups then exposed repeatedly to the sequences as described above. One group was tested on their memory for items within sequence 1 and sequence 2 and then tested on their memory for items between sequence 1 and sequence 2 , the same order in which the probe trials were administered to the mice in the experiment described above. Another group was first tested on memory for items between sequence 1 and sequence 2 and then tested on their memory for items within sequence 1 and sequence 2 , the opposite order as the groups described above. After the sequence probes, both groups were tested on novelty recognition probe trials.

Histology. After behavioral testing, all animals were given an overdose of sodium pentobarbital and perfused transcardially with $4 \%$ Formalin. The brains were removed and postfixed for $1 \mathrm{~h}$ in Formalin, and then cryoprotected in $30 \%$ sucrose solution, in $\mathrm{pH}$ 7.4 PBS. Coronal sections were cut $(40 \mu \mathrm{m})$ using a freezing microtome. Every section was mounted on gelatin-coated slides and dried overnight. Slides were soaked in xylenes and then run through a series of ethanol dehydrations, stained with cresyl violet, and then rehydrated. The extent of the lesion was determined using a light microscope to study the stained sections.

Four representative sections along the anteroposterior axis of the hippocampus ( $\mathrm{AP}+1.7,2.3,2.8$, and 3.08) were selected from the mouse brain atlas to determine hippocampal damage (Franklin and Paxinos, 1997). For each hippocampal lesion animal, the four brain slices most closely corresponding to the representative sections in the brain atlas were used. Three representative sections along the anteroposterior axis of the medial prefrontal cortex (AP - 2.58, 2.1, and 1.7) were selected from the mouse brain atlas to determine prefrontal damage (Franklin and Paxinos, 1997). For each prefrontal lesion animal, the three sections most closely corresponding to the representative sections in the brain atlas were used. Canvas 5.0 (Deneba Software; ACD Systems International) was used to calculate the percentage of tissue damage, which was defined as the amount of total damage divided by the total area of the brain in that section $\times 100$; the average of those values represented the lesion extent. Additional sections were studied under the light microscope to identify incidental damage outside the targeted regions and were reported in Results.

\section{Results}

\section{Histology}

In two animals, damage to the hippocampus was minimal, and these animals were removed from additional analyses. Otherwise, the NMDA infusions generally resulted in a substantial loss of cells within the hippocampal formation, including Ammon's horn, the dentate gyrus, and the subiculum (Fig. 2 A). An average of $45 \%$ of the hippocampus was damaged across animals, ranging from 8 to $77 \%$ total. The size of the lesion was not correlated with the extent of the behavioral deficit found $\left(r_{(8)}=-03.92, p=\right.$ $0.337)$. Two animals had partial damage to the thalamus. There was also incidental damage to multiple cortical areas above the infusion sites in both sham and hippocampal animals. The amount of damage in each cortical area is as follows: somatosensory cortex ( $10.8 \%$ in sham group, $18.88 \%$ in hippocampal lesion group; $\left.t_{(10)}=-1.892, p=0.088\right)$, motor cortex $(16.01 \%$ in sham group, $16.92 \%$ in hippocampal lesion group; $t_{(10)}=-0.07, p=$ $0.945)$, retrosplenial cortex $(25.26 \%$ in sham group, $21.75 \%$ in hippocampal lesion group; $\left.t_{(10)}=0.572, p=0.580\right)$, parietal cortex $(14.91 \%$ in sham group, $29.9 \%$ in hippocampal lesion group; $\left.t_{(10)}=-0.2337, p=0.042\right)$, and visual cortex $(8.92 \%$ in sham group, $27.54 \%$ in hippocampal lesion group; $t_{(10)}=$ $-4.130, p=0.033)$. Although the damage in the parietal and visual cortices was significantly greater in the hippocampal lesion group compared with the sham group, it is unlikely that slightly greater damage to these particular cortical areas could account for the pattern of impaired memory for odor order and spared odor recognition in this odor guided task.

Ibotenic acid infusions resulted in a substantial loss of cells within the medial wall of the prefrontal cortex, including the prelimbic and infralimbic cortices and dorsal cingulate gyrus (Fig. 2 B). An average of $43 \%$ of the medial prefrontal cortex was damaged across all animals, ranging from 12 to $81 \%$. Two animals had additional damage to the medial orbital cortex, and two animals had unilateral damage to the ventral and lateral orbital 
cortices. The size of the lesion was not correlated with the extent of the behavioral deficit found $\left(r_{(9)}=-0.46, p=0.172\right)$.

There were no significant differences in performance between sham-operated groups in which the infusions targeted the hippocampus and prefrontal cortex ("within-sequence" probe tests, $t_{(16)}=$ $-1.284, p=0.218$; "between-sequence" probe tests, $t_{(16)}=0.897, p=0.383$; "novelty" probe tests, $t_{(14)}=-0.256, p=$ $0.801)$; therefore, these two groups were combined and are collectively described as the "sham" group.

\section{Memory for items within each specific sequence}

Intact and sham-operated mice prefer to explore items that they had experienced earlier within each sequence, despite not being explicitly trained to judge the order of the items (sham-operated controls, $t_{(17)}$ $=6.637, p<0.001$; intact mice administered within-sequence probe trials first, $t_{(7)}=5.553, p<0.001$; intact mice administered between-sequence trials first, $t_{(7)}=3.556, p=0.003$ ) (Fig. $3 A, D$ ). These results indicate that sham-operated and intact mice remember the order in which they had experienced these sequences of odors. Importantly, the order in which probe trials were administered did not affect performance because mice given probe trials in opposite orders after training performed at equivalent levels on each type of probe trials (within-sequence probe trials, $t_{(14)}=-0.388, p=0.704$; between-sequence probe trials, $t_{(14)}=1.371, p=0.192$; novelty recognition probe trials, $\left.t_{(14)}=-0.624, p=0.543\right)$. Furthermore, without previous training on the sequences, naive mice did not exhibit a significant preference for the items occurring earlier within each sequence or between sequences; their scores accordingly did not differ from chance (within-sequence probe trials, $t_{(7)}=-0.540, p=0.598$; between-sequence probe trials, $t_{(7)}=$ $-0.185, p=0.85)$. Additionally, the scores of both groups of intact mice were significantly higher than the scores of the naive mice (one-way ANOVA, $F_{(2,23)}=6.851, p=0.005$; intact mice administered within-sequence probe trials first, $p=0.007$; intact mice administered between-sequence trials first, $p=0.003$ ) (Fig. $3 D$ ), indicating that the $5 \mathrm{~d}$ odor sequence presentations resulted in significant memory for the specific items in each sequence and does not reflect innate preferences.

Sham-operated subjects showed significantly greater preference indices than the operated groups (one-way ANOVA, $F_{(2,35)}=$ $17.008, p<0.001$ ) (Fig. 3A). Post hoc comparisons indicated that both animals with hippocampal damage $(p=0.001)$ and animals with prefrontal damage $(p<0.001)$ had lower preference scores than sham-operated animals, and the scores of animals with hippocampal lesions did not differ from those of the animals with medial prefrontal lesions $(p=0.284)$. Moreover, animals with damage to the hippocampus $\left(t_{(7)}=0.128, p=0.900\right)$ and those with damage to the medial prefrontal cortex $\left(t_{(9)}=-1.715, p=\right.$
0.103) did not exhibit preference scores that differed significantly from zero.

\section{Memory for order at different temporal lags between items within each sequence}

Six probe tests involving different odor comparisons were used to assess memory for odors within each sequence. These probe tests can further be segregated based on their temporal lags, that is, the number of items that occurred between odors when they were presented in the original sequences. We measured performance at four different lags, and our analyses separated these into "short" (0-1) and "long" (2-3) lags.

The three groups differed significantly in preference indices between short and long lags (two-way ANOVA, group, $F_{(2,71)}=$ 15.765, $p<0.001$; lag, $F_{(1,71)}=7.142, p=0.009$; group $\times$ lag interaction, $F_{(2,71)}=0.837, p=0.438$ ) (Fig. $3 A$ ). The group difference was highly significant at short lags (one-way ANOVA, $\left.F_{(2,35)}=13.113, p<0.001\right)$, and post hoc tests indicated that animals with hippocampal damage $(p=0.001)$, as well as animals with prefrontal damage $(p<0.001)$, had lower preference scores than sham-operated animals, but hippocampal and prefrontal groups did not differ from each other $(p=0.733)$. At long lags, there was also a significant difference among the three groups (one-way ANOVA, $F_{(2,35)}=4.429, p=0.02$ ), and post hoc tests indicated that animals with prefrontal damage $(p=0.024)$ and animals with hippocampal damage $(p=0.017)$ had signifi- 

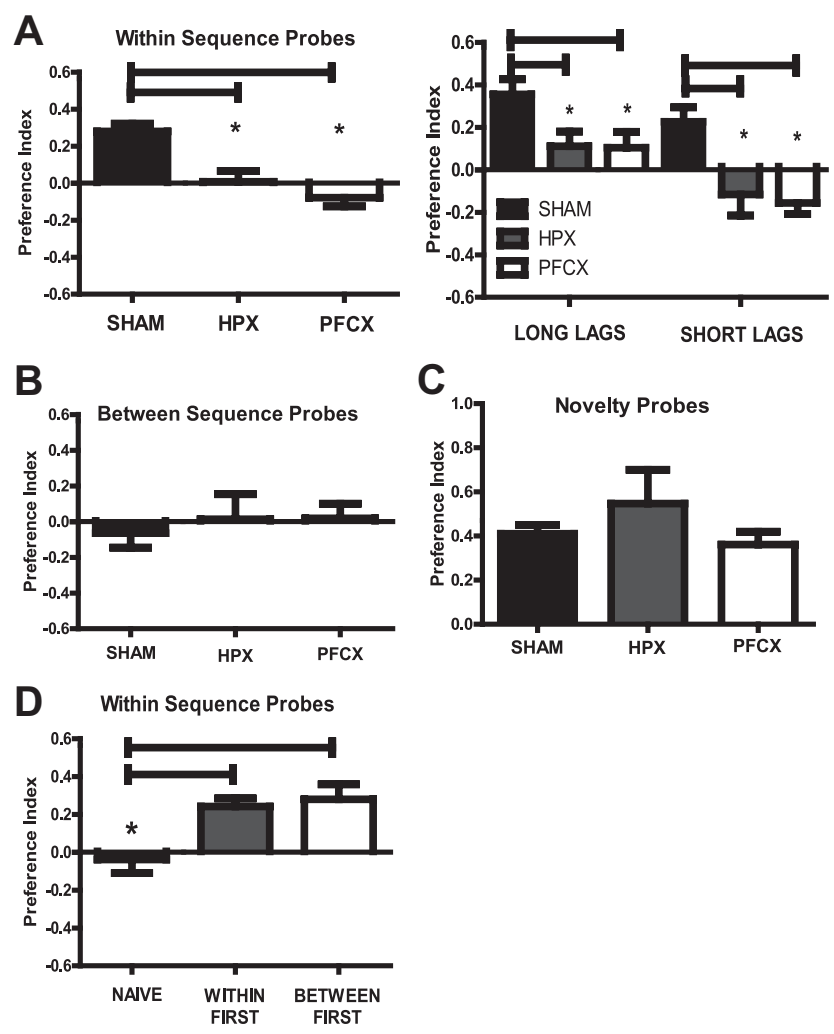

Figure 3. Performance of all three groups across the probe tests. $A$, Memory for the items was assessed by using a preference index ( \pm SEM). Animals with damage to the hippocampus or prefrontal cortex do not exhibit significant memory for items occurring earlier within each sequence; in addition, mice with lesions of the prefrontal cortex and hippocampus are impaired at all inner-item temporal lags relative to control mice. $\boldsymbol{B}$, Mice do not exhibit a significant preference for items occurring in sequence 1 compared with sequence 2 (preference index \pm SEM). C, All three groups exhibit a significant preference for novel versus familiar odors (preference index \pm SEM). $\boldsymbol{D}$, Intact mice administered sequence presentations across 5 d exhibited significant preferences for items occurring earlier within each series, but naive mice never exposed to these odors did not exhibit these preferences. The scores of the intact mice were significantly different from those of the naive mice, but both groups of intact mice demonstrated equivalent levels of preference regardless of the order in which they received the probe trial types. SHAM, Sham-operated mice; HPX, hippocampal lesion mice; PFCX, medial prefrontal lesion mice; NAIVE, mice never exposed to the odors before probe trials; WITHIN FIRST, intact mice administered within-sequence probe trials before within-sequence probe trials; BETWEEN FIRST, intact mice administered between-sequence probe trials before within-sequence probe trials. ${ }^{*} p<0.05$

cantly lower preference scores than sham-operated animals; however, the hippocampal and prefrontal groups did not differ from each other $(p=0.757)$.

Additional analyses of the direction of group preferences revealed a complex pattern of performance across short and long lags. The sham-operated group has a significant preference for earlier presented items at both short $\left(t_{(17)}=4.341, p<0.001\right)$ and long $\left(t_{(17)}=4.856, p<0.001\right)$ lags. At long lags, the mean scores of both lesion groups were above zero; this preference was not significant in animals with damage to the hippocampus $\left(t_{(7)}=0.638, p=0.534\right)$ or in animals with damage to the prefrontal cortex $\left(t_{(9)}=1.256, p=0.225\right)$. In contrast, at short lags, the mean scores of both lesion groups were below zero, indicating the opposite of normal preference; this effect was statistically significant in the prefrontal group $\left(t_{(9)}=-3.136, p=\right.$ $0.006)$ but not the hippocampal group $\left(t_{(7)}=-1.271, p=\right.$ $0.225)$. However, additional analyses revealed that, whereas sham-operated animals $\left(t_{(34)}=0.822, p=0.417\right)$ and hippocam- pal lesion animals $\left(t_{(14)}=-1.382, p=0.189\right)$ did not differ in performance between the short versus long lags, prefrontal lesion animals $\left(t_{(18)}=2.836, p=0.011\right)$ exhibited higher preference scores on long versus short lags.

\section{Memory for items across sequences}

To determine whether animals distinguished odors that appeared at widely separated times on the days in which sequences were presented, we administered probe tests comparing an odor from sequence 1 with an odor from sequence 2 . Neither shamoperated mice $\left(t_{(17)}=-0.795, p=0.432\right)$ nor animals with hippocampal $\left(t_{(7)}=0.098, p=0.923\right)$ or prefrontal $\left(t_{(9)}=0.219\right.$, $p=0.829)$ lesions exhibited any sign of preference or had preference indices that differed from zero (Fig. $3 B$ ). Accordingly, there was also no group difference (one-way ANOVA, $F_{(2,35)}=$ $0.26, p=0.773)$. Groups of intact mice also did not exhibit preferences for items in sequence 1 compared with items in sequence 2 (mice administered within-sequence probe trials first, $t_{(7)}=$ $1.349, p=0.19$; mice administered between-sequence trials first, $\left.t_{(7)}=-0.658, p=0.52\right)$. These findings suggest that the preferences exhibited on the within-sequence probe tests did not simply reflect greater interest in a less recently presented odor over a more recently presented odor on each day.

\section{Recognition of novel odors}

Animals with damage to the hippocampus or medial prefrontal cortex may have exhibited impairment on the probe tests for order memory secondary to an inability to remember the previously experienced odors. To examine this possibility, we administered novelty recognition tests, probe tests in which the animals were presented with an odor from sequence 1 or sequence 2 against a novel odor that was not presented in either sequence. All three groups showed significant preferences for the novel odor (sham-operated mice, $t_{(17)}=10.227, p<0.001$; hippocampal lesion mice, $t_{(5)}=3.549, p=0.005$; medial prefrontal lesion mice, $\left.t_{(9)}=6.17, p<0.001\right)$, and there was no difference in preference scores across the three groups (one-way ANOVA, $F_{(2,31)}=1.365, p=0.271$ ) (Fig. $3 C$ ). Groups of intact mice also demonstrated a significant preference for novel odors (mice administered within-sequence probe trials first, $t_{(7)}=8.377, p<$ 0.001 ; mice administered between-sequence probe trials first, $\left.t_{(7)}=5.845, p<0.001\right)$. Therefore, the impairment in memory for items within sequences is not attributable to the inability to recognize the odors.

Finally, there was no difference across groups in the total amount of time spent digging during probe tests for items within each sequence (one-way ANOVA, $F_{(2,415)}=2.109, p=0.123$ ), between each sequence (one-way ANOVA, $F_{(2,207)}=1.2, p=0.303$ ), or on novelty judgments (one-way ANOVA, $F_{(2,383)}=0.099, p=0.906$ ). Therefore, differences in preferences on the probe tests were not secondary to differences in activity level or interest (Table 1).

\section{Discussion}

In introducing the distinction between episodic and semantic memory, Tulving (1983) emphasized that episodic memories are characterized by the temporal organization of events within distinct experiences and contrasted this feature of episodic memory from the absence of temporal structure in semantic memories. This experiment was designed to determine whether mice can form representations of the temporal organization of events in distinct experiences. The results demonstrate for the first time that animals can indeed remember the order of events in specific sequential experiences without previous training on an order rule. Mice showed strong 


\begin{tabular}{|c|c|c|c|}
\hline & SHAM & HPX & $\mathrm{PFCX}$ \\
\hline \multicolumn{4}{|l|}{ Within sequence } \\
\hline Less recent odor & $4.22 \pm 0.39 \mathrm{~s}$ & $3.66 \pm 0.67 \mathrm{~s}$ & $4.08 \pm 0.53 \mathrm{~s}$ \\
\hline More recent odor & $2.40 \pm 0.28 \mathrm{~s}$ & $3.80 \pm 0.54 \mathrm{~s}$ & $4.25 \pm 0.41 \mathrm{~s}$ \\
\hline Preference index & $0.28 \pm 0.04$ & $0.01 \pm 0.06$ & $-0.08 \pm 0.05$ \\
\hline \multicolumn{4}{|l|}{ Between sequence } \\
\hline Odor from sequence 1 & $2.48 \pm 0.42 s$ & $3.04 \pm 0.76 \mathrm{~s}$ & $2.70 \pm 0.44 \mathrm{~s}$ \\
\hline Odor from sequence 2 & $2.21 \pm 0.27 \mathrm{~s}$ & $2.69 \pm 0.53 \mathrm{~s}$ & $3.29 \pm 0.63 \mathrm{~s}$ \\
\hline Preference index & $-0.06 \pm 0.08$ & $0.01 \pm 0.14$ & $0.02 \pm 0.08$ \\
\hline \multicolumn{4}{|l|}{ Novelty recognition } \\
\hline Novel odor & $4.22 \pm 0.45 \mathrm{~s}$ & $4.31 \pm 0.62 s$ & $4.51 \pm 0.50 \mathrm{~s}$ \\
\hline Familiar odor & $1.88 \pm 0.29 s$ & $2.18 \pm 0.77 \mathrm{~s}$ & $2.04 \pm 0.30 \mathrm{~s}$ \\
\hline Preference index & $0.41 \pm 0.04$ & $0.55 \pm 0.15$ & $0.36 \pm 0.06$ \\
\hline
\end{tabular}

SHAM, Sham-operated mice; HPX, hippocampal lesion mice; PFCX, medial prefrontal lesion mice.

preferential exploration of odors that they had experienced earlier within the same sequence of events but did not prefer an odor that they experienced hours earlier the same day in a different sequence. Therefore, their memory for order must be supported by a representation of the order of events specific to each sequence. We further demonstrated that this pattern of performance on within- and between-sequence comparisons is not dependent on the order in which these probe tests are presented. Our results also then confirm that the hippocampus and medial prefrontal cortex contribute to remembering order within specific experiences, whereas these areas are not essential to item recognition.

\section{Memory for the order of events in distinct sequences is not based on judgments of relative recency}

Previous behavioral studies have demonstrated that animals can remember the temporal order of serially presented odors (Fortin et al., 2002; Kesner et al., 2002) and the order of sequentially presented objects (Mitchell and Laiacona, 1998; Hannesson et al., 2004; Dere et al., 2005, 2007). However, to date, there has been no conclusive evidence as to whether animals make sequential memory judgments based on a representation of order within each specific experience or based on relative recency.

We first demonstrated that animals can successfully discriminate between the items occurring earlier compared with items occurring later within a specific sequence. In addition, there was no difference in memory for items that occurred farther apart compared with items that occurred closer together within that sequence. If order judgments were based on relative recency, we would have expected strong preferences in the comparison of odors that were presented $3 \mathrm{~h}$ apart across sequences than for comparisons that involved odors presented only seconds apart. Additionally, there was no difference between items at shorter compared with longer lags, suggesting that animals do not use recency information to make judgments about items within each sequence as well. Above-chance differential preferences on the between-sequence probe trials were not observed in either of our two intact groups or in either of our lesion groups; however, the sham group exhibited a significant preference for one of the three between-sequence probe trials. In other studies, we did not observe a significance preference on any between-sequence probes in two varieties of wild-type mice, mice lacking serine racemase (DeVito et al., 2010), or mice lacking the vasopressin 1B receptor (our unpublished findings), and all of these mice (except for the serine racemase knock-outs) showed the same strong withinsequence preferences as the mice in this study. Therefore, we view the absence of a between-sequence preference common among various groups of mice and judge the aberrant finding of one significant between-sequence preference as a statistical outlier that is not confirmed even among different types of betweensequence probes in our intact groups.

The present findings indicate that, across repeated sequence presentations, animals link the five odors within each complete sequence. Conversely, because the animals treat odors that appear in different sequences as unrelated, we conclude that mice viewed each sequence as a temporally organized experience that is distinct from the other sequence. We suggest that having been repeatedly rewarded for digging in the odors in a particular order may dispose the mice to spend more time searching for food in the odor for which digging had always previously been rewarded earlier; that is, they may have had a high expectancy of reward for odors that had regularly been rewarded earlier over those rewarded later. In addition, the abnormality of preferences in animals with hippocampal and prefrontal lesions suggests that, in the absence of prefrontal-hippocampal contributions, other brain systems lead to a very different pattern of choices.

\section{Contributions of the hippocampus and prefrontal cortex to sequence memory}

Memory for the order of events within each sequence was disrupted by damage to the hippocampus, confirming previous studies that have reported a critical role for the hippocampus in memory for sequential events (Fortin et al., 2002; Kesner et al., 2002), as well as in disambiguating items with overlapping elements (Agster et al., 2002; Ergorul and Eichenbaum, 2006; Kumaran and Maguire, 2006). Neuroimaging studies, as well as evidence from single-unit recordings, have also demonstrated that the hippocampus is activated during recall of the temporal order of life-like events (Gelbard-Sagiv et al., 2008; Lehn et al., 2009). Observations on ensemble neuronal activity have suggested that the hippocampus supports memory for specific sequences by the representation of an evolving temporal context for serial events within each sequence (Manns et al., 2007). Central to the findings presented here, Manns et al. (2007) reported that hippocampal neural ensembles do not code for the relative recency of sequential events nor do they tag items with their ordinal position within the sequence. Instead, when ensemble representations of the temporal context of each event distinguished experiences that occurred at different times across the sequence, memory for order was accurate. These results support the temporal context model of sequence memory, which proposes that events are bound within a sequence by a gradually changing temporal representation (Howard and Kahana, 2002).

Substantial evidence also supports a role for the prefrontal cortex in memory for the temporal order of events (Mitchell and Laiacona, 1998; Fuster, 2001; Hannesson et al., 2004; Barker et al., 2007). Electrophysiological data collected from animals performing a task similar to that in this study suggest that an additional region of the prefrontal cortex plays a role in anticipating sequential events (Ramus et al., 2007). In that experiment, animals were presented repetitively with a series of eight odors as the activity of orbitofrontal neurons was monitored. In well-trained animals, neurons in orbitofrontal cortex fired selectively during the sampling of particular odors, and some cells exhibited odor-selective firing 200-400 ms before odor onset. Furthermore, damage to the hippocampus abolished this anticipatory firing pattern, suggesting that input from the hippocampus provides important information about the anticipation of sequential odors. In a study that involved recordings in monkeys performing a temporal ordering task, lateral prefrontal cortex cells fired selectively to items 
within a specific sequence, and this activity predicted behavioral judgments about order (Ninokura et al., 2003; Averbeck and Lee, 2007). These findings suggest that the prefrontal cortex may establish representations of orderly events and may retrieve these representations guided by input from the hippocampus.

\section{Hippocampal-cortical circuitry supports memory for the order of items within a sequence but does not support item recognition}

There is a current controversy as to how the hippocampal-cortical circuitry supports memory strength judgments (Eichenbaum et al., 2007; Squire et al., 2007; Shrager et al., 2008; Diana et al., 2010). Recent findings have suggested that both the hippocampus and prefrontal cortex play important roles in the recollection component of recognition memory but do not contribute to familiarity (Fortin et al., 2004; Farovik et al., 2008). Additional studies using object recognition have found that these structures do not contribute to the detection of novel compared with familiar items but instead are recruited when familiar items are combined in novel ways, such as when associations between these items must be formed (Dudchenko et al., 2000; Hannesson et al., 2004; Jenkins et al., 2004; Aggleton and Brown, 2005). Despite a severe impairment in order memory, animals with damage to the hippocampus or prefrontal cortex successfully recognized each of the items in the two sequences.

The current findings provide a novel method for evaluating sequence memory and demonstrate that animals can remember the order of events within specific experiences, and, in a parallel study, this test was useful in distinguishing a cognitive impairment in a murine model of schizophrenia (DeVito et al., 2010). In addition, the present results resolve an important issue in the development of animal models of episodic memory, demonstrating that normal animals can remember specific sequences without relying on judgments about the mere relative recency of temporally separated events and that hippocampal-cortical circuitry supports the temporal organization of memories.

\section{References}

Aggleton JP, Brown MW (2005) Contrasting hippocampal and perirhinal cortex function using immediate early gene imaging. Q J Exp Psychol B 58:218-233.

Agster KL, Fortin NJ, Eichenbaum H (2002) The hippocampus and disambiguation of overlapping sequences. J Neurosci 22:5760-5768.

Averbeck BB, Lee D (2007) Prefrontal neural correlates of memory for sequences. J Neurosci 27:2204-2211.

Barker GR, Bird F, Alexander V, Warburton EC (2007) Recognition memory for objects, place, and temporal order: a disconnection analysis of the role of the medial prefrontal cortex and perirhinal cortex. J Neurosci 27:2948-2957.

Bunsey M, Eichenbaum H (1996) Conservation of the hippocampal memory function in rats and humans. Nature 379:255-257.

Chiba AA, Kesner RP, Gibson CJ (1997) Memory for the temporal order of new and familiar spatial location sequences: role of the medial prefrontal cortex. Learn Mem 4:311-317.

Clayton NS, Dickinson A (1998) Episodic-like memory during cache recovery by scrub jays. Nature 395:272-274.

Dere E, Huston J, De Souza Silva M (2005) Episodic-like memory in mice: simultaneous assessment of object, place and temporal order memory. Brain Res Brain Res Prot 16:10-19.

Dere E, Huston JP, De Souza Silva MA (2007) The pharmacology, neuroanatomy and neurogenetics of one-trial object recognition in rodents. Neurosci Biobehav Rev 31:673-704.

DeVito L, Balu D, Kanter B, Lykken C, Basu A, Coyle J, Eichenbaum H (2010) Serine racemase deletion disrupts memory for order and alters cortical den- dritic morphology. Genes Brain Behav. Advance online publication. Retrieved January 24, 2011. doi:10.1111/j.1601-183X.2010.00656.x.

Diana RA, Yonelinas AP, Ranganath C (2010) Medial temporal lobe activity during source retrieval reflects information type, not memory strength. J Cogn Neurosci 22:1808-1818.

Dudchenko PA, Wood ER, Eichenbaum H (2000) Neurotoxic hippocampal lesions have no effect on odor span and little effect on odor recognition but produce significant impairments on spatial span, recognition, and alternation. J Neurosci 20:2964-2977.

Eichenbaum H (2004) Hippocampus: cognitive processes and neural representations that underlie declarative memory. Neuron 44:109-120.

Eichenbaum H, Yonelinas AP, Ranganath C (2007) The medial temporal lobe and recognition memory. Annu Rev Neurosci 30:123-152.

Ergorul C, Eichenbaum H (2006) Essential role of the hippocampal formation in rapid learning of higher-order sequential associations. J Neurosci 26:4111-4117.

Farovik A, Dupont LM, Arce M, Eichenbaum H (2008) Medial prefrontal cortex supports recollection, but not familiarity, in the rat. J Neurosci 28:13428-13434.

Fortin NJ, Agster KL, Eichenbaum HB (2002) Critical role of the hippocampus in memory for sequence of events. Nat Neurosci 5:458-462.

Fortin NJ, Wright SP, Eichenbaum H (2004) Recollection-like memory retrieval in rats is dependent on the hippocampus. Nature 431:188-191.

Franklin K, Paxinos G (1997) The mouse brain in stereotaxic coordinates. San Diego: Academic.

Fuster JM (2001) The prefrontal cortex-an update: time is of the essence. Neuron 30:319-333.

Gelbard-Sagiv H, Mukamel R, Harel M, Malach R, Fried I (2008) Internally generated reactivation of single neurons in human hippocampus during free recall. Science 322:96-101.

Hannesson DK, Vacca G, Howland JG, Phillips AG (2004) Medial prefrontal cortex is involved in spatial temporal order memory but not spatial recognition memory in tests relying on spontaneous exploration in rats. Behav Brain Res 153:273-285.

Howard M, Kahana M (2002) A distributed representation of temporal context. J Math Psych 46:269-299.

Jenkins TA, Amin E, Pearce JM, Brown MW, Aggleton JP (2004) Novel spatial arrangements of familiar visual stimuli promote activity in the rat hippocampal formation but not the parahippocampal cortices: a $c$-fos expression study. Neuroscience 124:43-52.

Kesner RP, Gilbert PE, Barua LA (2002) The role of the hippocampus in memory for the temporal order of a sequence of odors. Behav Neurosci 116:286-290.

Kumaran D, Maguire EA (2006) The dynamics of hippocampal activation during encoding of overlapping sequences. Neuron 49:617-629.

Lehn H, Steffenach HA, van Strien NM, Veltman DJ, Witter MP, Håberg AK (2009) A specific role of the human hippocampus in recall of temporal sequences. J Neurosci 29:3475-3484.

Manns JR, Howard MW, Eichenbaum H (2007) Gradual changes in hippocampal activity support remembering the order of events. Neuron 56:530-540

Mitchell JB, Laiacona J (1998) The medial frontal cortex and temporal memory: tests using spontaneous exploratory behaviour in the rat. Behav Brain Res 97:107-113.

Ninokura Y, Mushiake H, Tanji J (2003) Representation of the temporal order of visual objects in the primate lateral prefrontal cortex. J Neurophysiol 89:2868-2873.

Ramus SJ, Davis JB, Donahue RJ, Discenza CB, Waite AA (2007) Interactions between the orbitofrontal cortex and the hippocampal memory system during the storage of long-term memory. Ann NY Acad Sci 1121:216-231.

Shrager Y, Kirwan CB, Squire LR (2008) Activity in both hippocampus and perirhinal cortex predicts the memory strength of subsequently remembered information. Neuron 59:547-553.

Squire LR, Wixted JT, Clark RE (2007) Recognition memory and the medial temporal lobe: a new perspective. Nat Rev Neurosci 8:872-883.

Tulving E (1983) Elements of episodic memory. London: Oxford UP.

Tulving E, Markowitsch HJ (1998) Episodic and declarative memory: role of the hippocampus. Hippocampus 8:198-204. 